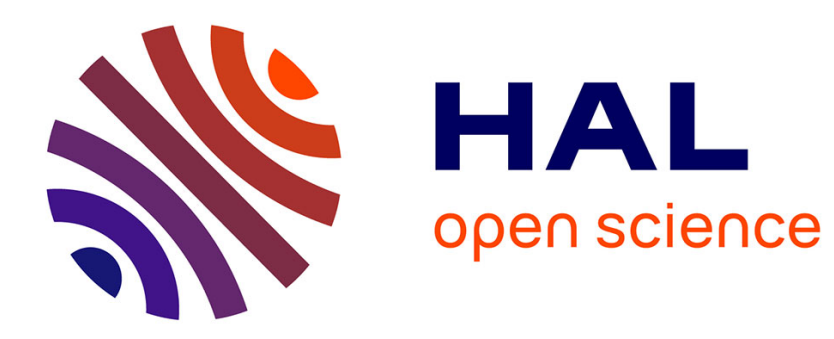

\title{
Adaptive step rotation in biped walking
}

Néstor Bohórquez, Pierre-Brice Wieber

\section{To cite this version:}

Néstor Bohórquez, Pierre-Brice Wieber. Adaptive step rotation in biped walking. IROS 2018 IEEE/RSJ International Conference on Intelligent Robots and Systems, Oct 2018, Madrid, Spain. pp.720-725, 10.1109/IROS.2018.8594431 . hal-01957332

\section{HAL Id: hal-01957332 \\ https://hal.science/hal-01957332}

Submitted on 17 Dec 2018

HAL is a multi-disciplinary open access archive for the deposit and dissemination of scientific research documents, whether they are published or not. The documents may come from teaching and research institutions in France or abroad, or from public or private research centers.
L'archive ouverte pluridisciplinaire HAL, est destinée au dépôt et à la diffusion de documents scientifiques de niveau recherche, publiés ou non, émanant des établissements d'enseignement et de recherche français ou étrangers, des laboratoires publics ou privés. 


\title{
Adaptive step rotation in biped walking
}

\author{
Néstor Bohórquez and Pierre-Brice Wieber
}

\begin{abstract}
We want to enable the robot to reorient its feet in order to face its direction of motion. Model Predictive Control schemes for biped walking usually assume fixed feet rotation since adapting them online leads to a nonlinear problem. Nonlinear solvers do not guarantee the satisfaction of nonlinear constraints at every iterate and this can be problematic for the real-time operation of robots. We propose to define safe linear constraints that are always inside the intersection of the nonlinear constraints. We make simulations of the robot walking on a crowd and compare the performance of the proposed method with respect to the original nonlinear problem solved as a Sequential Quadratic Program.
\end{abstract}

\section{INTRODUCTION}

When walking in a dynamic environment, a biped robot can need to change its velocity in order to avoid collisions, what needs to be coordinated with changes in orientation. This is a non-trivial problem to solve and we can observe that humans switch between holonomic (walking sideways) and nonholonomic locomotion (changing orientation) depending on the situation and goals [1] [2]. In this paper, we want the robot to face its direction of motion because it walks faster forwards than sideways.

Turning while walking has been addressed in many different ways [3] [4] [5] [6] [7] [8] [9] but never guaranteeing that all kinematic and dynamic constraints are properly satisfied. We can make sure to generate walking motions that satisfy all constraints with a Model Predictive Control (MPC) approach [10] [11]. This is usually done by decoupling the computation of the motion of the Center of Mass (CoM) and the Center of Pressure (CoP) from the computation of the motion of the rest of the body. This is a very effective method that has been used in a wide variety of robots [12] [13] [14].

However, rotations are usually decided in advance [15] [16] because they introduce nonlinearities in the kinematic and dynamic constraints [17]. Nonlinear constraints are usually addressed iteratively using a Newton method and their satisfaction is not guaranteed until convergence. This is incompatible with real time control where we want to have a feasible solution (satisfying all constraints and safely applicable to the robot) at a specified time.

We want to make sure to have a feasible solution after a single Newton iteration. We propose to use a common method to handle nonlinear constraints: we define new, safe linear constraints that are always inside the intersection of the original nonlinear constraints. This method is useful when

\footnotetext{
Univ. Grenoble Alpes, Inria, r CNRS, Grenonestor-alonso.bohorquez-dorantedinria.fr, pierre-brice.wiebereinria.fr.
}

adapting the duration of steps [18] and the vertical motion of the CoM [19] [20]. It has also been used for the adaptation of the rotation of the steps [21] but, in comparison, we propose constraints that generate less restrictive motions.

Using a simulation of the robot walking in a crowd as a test bed, we propose to investigate how the feasibility of the problem and the behaviour of the robot vary with the number of Newton iterations in comparison with a standard Sequential Quadratic Programming (SQP) method.

We proceed as follows: we present the model that relates the motion of the CoM with the position of the CoP in Section II. We define a set of dynamic and kinematic constraints of the robot that are linear and safe with respect to rotations in Section III. Section IV formulates the Optimal Control Problem (OCP) that we need to solve and Section V shows some numerical results that demonstrate the capabilities of the controller.

\section{DYNAMIC MODEL}

We model the motion of the CoM of the robot as a triple integrator with the $\mathrm{CoP}$ as an output of the system [22]

$$
\begin{aligned}
x^{+} & =\left[\begin{array}{ccc}
1 & \tau & \tau^{2} / 2 \\
0 & 1 & \tau \\
0 & 0 & 1
\end{array}\right] x+\left[\begin{array}{c}
\tau^{3} / 6 \\
\tau^{2} / 2 \\
\tau
\end{array}\right] \dddot{c}, \\
p & =\left[\begin{array}{lll}
1 & 0 & -h / g
\end{array}\right] x .
\end{aligned}
$$

In here, $c$ is the position of the CoM, the jerk $\dddot{c}$ is the input of the system, $x=(c, \dot{c}, \ddot{c})$ and $x^{+}=\left(c^{+}, \dot{c}^{+}, \ddot{c}^{+}\right)$ are two consecutive states, $\tau>0$ is the sampling time, $p$ is the position of the CoP, $h$ is the height of the CoM and $g$ is the norm of the vector of gravity. We assume $h$ to be constant [23] [17] [22].

\section{SAFE LINEAR CONSTRAINTS}

\section{A. Constraint on the steps}

We want to avoid leg-crossing in the sequence of step positions $\left(s_{1}, \ldots, s_{J}\right)$ and step orientations $\left(\theta_{1}, \ldots, \theta_{J}\right)$ that we compute during the preview horizon. Each foot is a rectangle of length $l$ and width $w$. We constrain the position of the $(j+1)^{t h}$ step $s_{j+1}$ to the half-space that starts at a distance $w$ from $s_{j}$ in the direction given by the rotated normalized vector $R\left(\theta_{j}\right) \hat{n}_{j}$.

$$
w \leq\left(R\left(\theta_{j}\right) \hat{n}_{j}\right)^{T} s_{j+1}-s_{j} .
$$

The rotation matrix $R\left(\theta_{j}\right)$ introduces nonlinearities. We want to define a new set of constraints on the steps that are linear and safe with respect to rotations when we restrict $\theta_{j}$ to an interval $\left[\underline{\theta}_{j}, \bar{\theta}_{j}\right]$. 


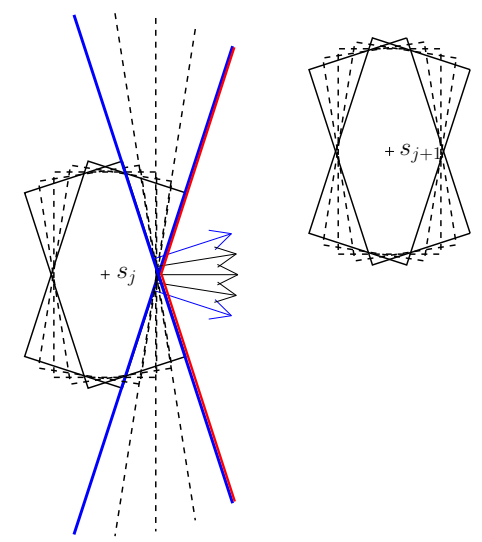

Fig. 1: Constraints on the steps. The solid rectangles on the left represent the foot $s_{j}$ rotated at angles $\underline{\theta}_{j}$ and $\bar{\theta}_{j}$; the dotted rectangles represent various rotations in-between. The long lines at the border of these rectangles along with the arrows perpendicular to them define the half-spaces of permitted positions of the foot $s_{j+1}$. The minimal distance $d$ between the center of the feet is adjusted according to (5).

In Figure 1 the constraint (2) is illustrated for rotations that satisfy $|\Delta \theta| \leq \Delta \theta_{c}$, where

$$
\Delta \theta_{c}=2 \arcsin (w / l),
$$

The safe set with respect to the rotations of the foot $s_{j}$ (shown in red) is the intersection of the half-spaces defined with $\underline{\theta}_{j}$ and $\bar{\theta}_{j}$ (shown in blue):

$$
\begin{aligned}
& d \leq\left(R\left(\underline{\theta}_{j}\right) \hat{n}_{j}\right)^{T} s_{j+1}-s_{j}, \\
& d \leq\left(R\left(\bar{\theta}_{j}\right) \hat{n}_{j}\right)^{T} s_{j+1}-s_{j} .
\end{aligned}
$$

The minimal distance $d$ that separates the centers of the feet $s_{j}$ and $s_{j+1}$ is adapted with $\Delta \theta$ to avoid collisions between the rotated feet:

$$
d=w+1 / 2(l-w \tan (\Delta \theta / 2)) \sin (\Delta \theta) .
$$

\section{B. Constraint on the CoP}

Due to the unilaterality of the contact forces with the ground, the CoP $p$ can only reside inside the support polygon of the robot:

$$
\left[\begin{array}{c}
-l / 2 \\
-w / 2
\end{array}\right] \leq R\left(\theta_{j}\right)^{T} p-s_{j} \leq\left[\begin{array}{c}
l / 2 \\
w / 2
\end{array}\right]
$$

The rotation matrix $R\left(\theta_{j}\right)$ changes the orientation of the foot by $\theta_{j}$.

Figure 2 shows in red that, as we vary $\theta_{j}$, the area inside the foot that remains safe with respect to rotations is the intersection of: 1) the foot rotated $\underline{\theta}_{j}, 2$ ) the foot rotated $\left.\bar{\theta}_{j}, 3\right)$ the circle centered at $s_{j}$ with radius $l / 2$, plus a more complicated operation with the circle centered at $s_{j}$ with radius $w / 2$.

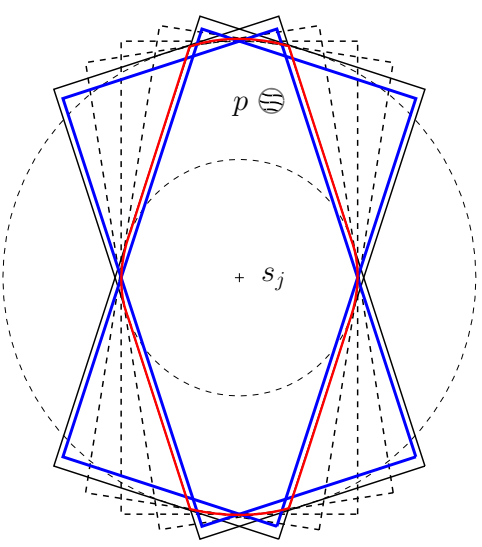

Fig. 2: Constraints on the CoP. The solid and dotted rectangles in black represent the foot $s_{j}$ rotated at various angles between $\underline{\theta}_{j}$ and $\bar{\theta}_{j}$. The red line is the perimeter of the safe nonlinear constraint. The blue lines are the perimeters of the reduced areas inside the foot rotated at angles $\underline{\theta}_{j}$ and $\bar{\theta}_{j}$.

Unfortunately, circular constraints are nonlinear. We propose to avoid them by reducing the size of the permitted area for the CoP some $\Delta w$ and $\Delta l$ given by

$$
\Delta w=w(1-\cos (\Delta \theta / 2)), \quad \Delta l=l(1-\cos (\Delta \theta / 2)) .
$$

The original set can then be approximated by the intersection of the reduced-size foot rotated $\underline{\theta}_{j}$ and $\bar{\theta}_{j}$ :

$$
\begin{aligned}
& {\left[\begin{array}{c}
-\hat{l} / 2 \\
-\hat{w} / 2
\end{array}\right] \leq R\left(\underline{\theta}_{j}\right)^{T} p-s_{j} \leq\left[\begin{array}{c}
\hat{l} / 2 \\
\hat{w} / 2
\end{array}\right],} \\
& {\left[\begin{array}{c}
-\hat{l} / 2 \\
-\hat{w} / 2
\end{array}\right] \leq R\left(\bar{\theta}_{j}\right)^{T} p-s_{j} \leq\left[\begin{array}{c}
\hat{l} / 2 \\
\hat{w} / 2
\end{array}\right],}
\end{aligned}
$$

shown in blue in the figure, where

$$
\hat{l}=l-\Delta l, \quad \hat{w}=w-\Delta w .
$$

\section{Constraint on the CoM}

Since the length of the legs is limited, the position of the CoM with respect to the foot on the ground has to satisfy:

$$
\left[\begin{array}{c}
-L / 2 \\
0
\end{array}\right] \leq R\left(\theta_{j}\right)^{T} c-s_{j} \leq\left[\begin{array}{c}
L / 2 \\
W
\end{array}\right]
$$

which defines a rectangle of permitted positions of the CoM with respect to $s_{j}$. This rectangle has width $W$, length $L$ and orientation $\theta_{j}$ set by the rotation matrix $R\left(\theta_{j}\right)$.

We analyze how the original constraint evolves with $\theta_{j}$ in Figure 3. The safe nonlinear set (shown in red) is the intersection of the rectangle rotated by $\underline{\theta}_{j}$ and $\bar{\theta}_{j}$ plus a more complicated operation with the circle of radius $W$ centered at $s_{j}$. As before, we can approximate this set to the intersection of

$$
\begin{aligned}
& {\left[\begin{array}{c}
-L / 2 \\
0
\end{array}\right] \leq R\left(\underline{\theta}_{j}\right)^{T} c-s_{j} \leq\left[\begin{array}{c}
L / 2 \\
\hat{W}
\end{array}\right]} \\
& {\left[\begin{array}{c}
-L / 2 \\
0
\end{array}\right] \leq R\left(\bar{\theta}_{j}\right)^{T} c-s_{j} \leq\left[\begin{array}{c}
L / 2 \\
\hat{W}
\end{array}\right],}
\end{aligned}
$$




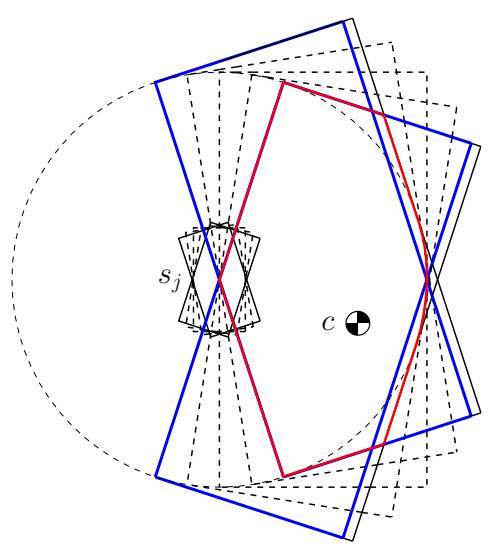

Fig. 3: Constraint on the CoM. The permitted area for the CoM has width $W$ and length $L$.

by reducing the width of the rectangle of permitted positions of the CoM some $\Delta W$ given by:

$$
\Delta W=W(1-\cos (\Delta \theta / 2)) .
$$

and $\hat{W}=W-\Delta W$. The resulting set is shown in blue in the figure.

\section{Constraint on collision avoidance}

In order to avoid collisions, we constrain the position of the CoM to be at a distance no shorter than $D$ from each person $m_{k}$ in the crowd:

$$
\forall k:\left\|c-m_{k}\right\|_{2} \geq D,
$$

as in [24]. The rotation of the feet does not affect directly this constraint.

\section{E. Constraint on capturability}

Given a preview horizon of $N$ samples, we achieve 0 step capturability (the ability to stop the motion of the CoM without having to make any extra step [25]) at the end of this horizon by making the last Capture Point sample $\xi_{N}$, defined as

$$
\xi_{N}=\left[\begin{array}{lll}
1 & \sqrt{h / g} & 0
\end{array}\right] x_{N},
$$

reside inside the perimeter of the last step:

$$
\left[\begin{array}{c}
-l / 2 \\
-w / 2
\end{array}\right] \leq R\left(\theta_{J}\right)^{T} \xi_{N}-s_{J} \leq\left[\begin{array}{l}
l / 2 \\
w / 2
\end{array}\right]
$$

Just like we did with the CoP, we can define a safe linear version of (15) as:

$$
\begin{aligned}
& {\left[\begin{array}{c}
-\hat{l} / 2 \\
-\hat{w} / 2
\end{array}\right] \leq R\left(\underline{\theta}_{J}\right)^{T} \xi_{N}-s_{J} \leq\left[\begin{array}{c}
-\hat{l} / 2 \\
-\hat{w} / 2
\end{array}\right],} \\
& {\left[\begin{array}{c}
-\hat{l} / 2 \\
-\hat{w} / 2
\end{array}\right] \leq R\left(\bar{\theta}_{J}\right)^{T} \xi_{N}-s_{J} \leq\left[\begin{array}{c}
-\hat{l} / 2 \\
-\hat{w} / 2
\end{array}\right],}
\end{aligned}
$$

with the appropriate reduction of the size of the foot described in (7).
TABLE I: Parameters of biped robot

\begin{tabular}{cccc}
\hline Parameter & Symbol & Value & Unit \\
\hline Height of & $h$ & 0.80 & $\mathrm{~m}$ \\
CoM (1) & & $0.25 \times 0.125$ & $\mathrm{~m} \times \mathrm{m}$ \\
Feet dim. (6) & $l \times w$ & $0.30 \times 0.30$ & $\mathrm{~m} \times \mathrm{m}$ \\
Leg stride (10) & $L \times W$ & 1 & $\mathrm{~m}$ \\
Safety & $D$ & 4 & $\mathrm{~m}$ \\
distance (13) & $R$ & 18 & samples $\left.^{-1}, \mathrm{~m}^{-1}\right)$ \\
Radius of FoV & $N$ & $(0.5,0)$ & $\left(\mathrm{m}^{-1}\right)$ \\
Horizon length & $\dot{c}_{r e f}$ & &
\end{tabular}

\section{Model Predictive Control for Walking}

We define objectives to describe the tasks we want the robot to perform: 1) move the $\mathrm{CoM}$ at a reference velocity $\left.\dot{c}_{\text {ref }}, 2\right)$ keep the CoP at the center of the foot for improved balance, 3) minimize the jerk of the CoM for smoothness of its motion and regularization, 4) align the feet with the direction where the CoM is moving 5) stop the robot at the end of the horizon to ensure capturability. In summary, we consider the following objectives:

$$
g=\left[\begin{array}{c}
\dot{c}-\dot{c}_{r e f} \\
p-s_{j} \\
\dddot{c} \\
\left(\cos \left(\theta_{j}\right), \sin \left(\theta_{j}\right)\right) \times \dot{c} \\
p_{N}-\xi_{N}
\end{array}\right]=0
$$

in the following OCP:

$$
\begin{aligned}
\underset{\left\{\dddot{c}_{i}\right\},\left\{s_{j}\right\},\left\{\theta_{j}\right\}}{\operatorname{minimize}} & \sum_{i=1}^{N}\left\|g\left(\dddot{c}_{i}, s_{j}, \theta_{j}\right)\right\|_{Q}^{2} \\
\text { subject to } & (4),(8),(11),(13), \\
& \theta_{j} \in\left[\underline{\theta}_{j}, \bar{\theta}_{j}\right],
\end{aligned}
$$

where $\mathrm{Q}$ is a diagonal matrix of weights.

The function $g$ is nonlinear with respect to $\theta_{j}$ so we apply a Newton method and consider $I$ successive linearizations. After each iteration we adapt the interval of rotations for the $j^{t h}$ foot $\left[\underline{\theta}_{j}, \bar{\theta}_{j}\right]$ based on the previous optimal rotation of the $(j-1)^{t h}$ foot $\theta_{j-1}^{*}$ :

$$
\underline{\theta}_{j}=\theta_{j-1}^{*}-\frac{\Delta \theta}{2}, \quad \bar{\theta}_{j}=\theta_{j-1}^{*}+\frac{\Delta \theta}{2} .
$$

\section{Simulation RESUlts}

We evaluate the performance of our controller by simulating a robot and a crowd walking in opposite directions. As in [24], we assume that: people walk at constant velocities, they do not try to avoid the robot and we disregard collisions among them. The parameters of the robot are specified in Table I.

We use the notion of a Field of View (FoV): the maximal distance around the robot at which it is capable of perceiving persons/objects. We do not consider occlusions in perception, making the robot aware of the current position and velocity of everybody within the FoV. 
TABLE II: Parameters of scenario A

\begin{tabular}{cccc}
\hline Parameter & Symbol & Value & Unit \\
\hline Size of crowd & $M$ & 20 & persons \\
Vel. of the crowd & $\dot{m}_{k}^{x}$ & -0.5 & $\mathrm{~m} \cdot \mathrm{s}^{-1}$ \\
Length of interval & $\dot{m}_{k}^{y}$ & $\mathcal{N}(0,0.5)$ & $\mathrm{m} \cdot \mathrm{s}^{-1}$ \\
rotations & $\Delta \theta$ & 30 & degrees \\
Number of & $I$ & 1 & - \\
Newton iterations & & & \\
\hline
\end{tabular}

\section{A. Typical behaviour}

Figure 4 shows the results obtained in a typical simulation. The parameters of the controller and the crowd for this scenario are specified in Table II. The figure shows all the steps made by the robot during the simulation. The robot changes its orientation several times to avoid the people in the crowd. Once the robot overcomes the crowd, it changes its orientation back to 0 degrees to more efficiently track its reference velocity $\dot{c}_{r e f}$.

\section{B. Comparison with standard SQP}

We make a comparison between our proposed method to handle nonlinear constraints and a standard SQP. The proposed SQP solves the following nonlinear problem with nonlinear constraints:

$$
\begin{aligned}
\underset{\left\{\dddot{c}_{i}\right\},\left\{s_{j}\right\},\left\{\theta_{j}\right\}}{\operatorname{minimize}} & \sum_{i=1}^{N}\left\|g\left(\dddot{c}_{i}, s_{j}, \theta_{j}\right)\right\|_{Q}^{2} \\
& (2),(6),(10),(13),(15) \\
\text { subject to } & \theta_{j} \in\left[\underline{\theta}_{j}, \bar{\theta}_{j}\right] .
\end{aligned}
$$

We make 600 simulations (300 with our method and 300 with SQP) of the robot walking in randomly generated crowds according to the parameters shown in Table III. We generate and store 25 different crowds with speed $\dot{m}_{k}^{x}$ that only differ in the initial positions $\left\{m_{k}\right\}$ and speeds $\left\{\dot{m}_{k}^{y}\right\}$ of each person. Their initial positions vary uniformly over an area of $10 \times 8\left[\mathrm{~m}^{2}\right]$ while their speeds $\left\{\dot{m}_{k}^{y}\right\}$ follow a normal distribution $\mathcal{N}(0,0.5)$. During simulations, we test the performance of the controller with each $\Delta \theta$ and each $I$ against each of the 25 crowds generated for $\dot{m}_{k}^{x}$. Simulations last 20 [s] or until a collision or a kinematic/dynamic failure occurs.

Results are shown in Figure 5. We take as base case $\Delta \theta=0$. When we solve it with either method the results are: less than $1.5 \%$ of all Newton iterations are infeasible, $12 \%$ of simulations end up in a previewed collision and $0 \%$ of simulations end up in a nonpreviewed kinematic or dynamic failure. When the OCP becomes infeasible we can continue executing the last computed trajectory until it becomes feasible again. If this does not happen, the last computed trajectory ensures that the robot will come to a stop before any collision happens, as in [24].

When we solve the nonlinear problem using SQP with only one Newton iteration we have that, for all $\Delta \theta>0$,
TABLE III: Parameters of scenario B

\begin{tabular}{cccc}
\hline Parameter & Symbol & Value & Unit \\
\hline Size of crowd & $M$ & 16 & persons \\
Vel. of the crowd & $\dot{m}_{k}^{x}$ & -0.5 & $\mathrm{~m} \cdot \mathrm{s}^{-1}$ \\
Length of interval & $\dot{m}_{k}^{y}$ & $\mathcal{N}(0,0.5)$ & $\mathrm{m} \cdot \mathrm{s}^{-1}$ \\
sampling time & $\Delta \theta$ & $\{0,15,30,45\}$ & $\mathrm{deg}$ \\
Number of & $\mathrm{I}$ & $\{1,3,5\}$ & - \\
Newton iterations & & & \\
\hline
\end{tabular}

all simulations end up in nonpreviewed kinematic/dynamic failures. This means that the solution given by a single Newton iteration does not satisfy the nonlinear constraints. With higher values of $I$ the failure rate reduces dramatically and the collision rate improves with respect to the base case. However, the failure rate increases with the length of the interval of rotations because the linearizations become less reliable.

When we solve the problem with safe linear constraints, the problem is feasible all over the interval of rotations, therefore, the failure rate is zero. However, the augmented constraints reduce the mobility of the robot and collision avoidance is, in general, not as good as with an SQP but still equal or better than the base case. The number of infeasible iterations is very similar to what we obtain with an SQP.

The best choice for SQP seems to be $I=3$ iterations with $\Delta \theta=15[\mathrm{deg}]$. Observe that the same performance can be obtained with the method of safe linear constraints with just $I=1$ and $\Delta \theta=15[\mathrm{deg}]$.

\section{Computational complexity}

The OCP (20) contains 42 decision variables. The number of constraints is proportional to the number of obstacles in the FoV which is less or equal to $M$. In our simulations the total number of constraints oscillates between 300 and 600 . A single iteration of this problem is solved on average in $0.51[\mathrm{~ms}]$ on a laptop with a $3[\mathrm{GHz}]$ Intel Core i7 CPU.

\section{CONCLUSIONS}

We presented a method to adapt the rotation of the steps of the biped robot to allow it to face its direction of motion. Rotations on the feet introduced nonlinear constraints in the formulation of the controller. We guarantee the satisfaction of these nonlinear constraints at every Newton iteration. To do so, we wrote linear constraints that are safe with respect to a given interval of rotations. We made simulations to showcase the real-time performance of the controller and compared it with a standard SQP.

\section{ACKNOWLEDGMENTS}

This work has been funded by the PSPC Romeo 2 project and EU H2020 Comanoid Research and Innovation Action (RIA). 


\section{Biped robot walking in a crowd}

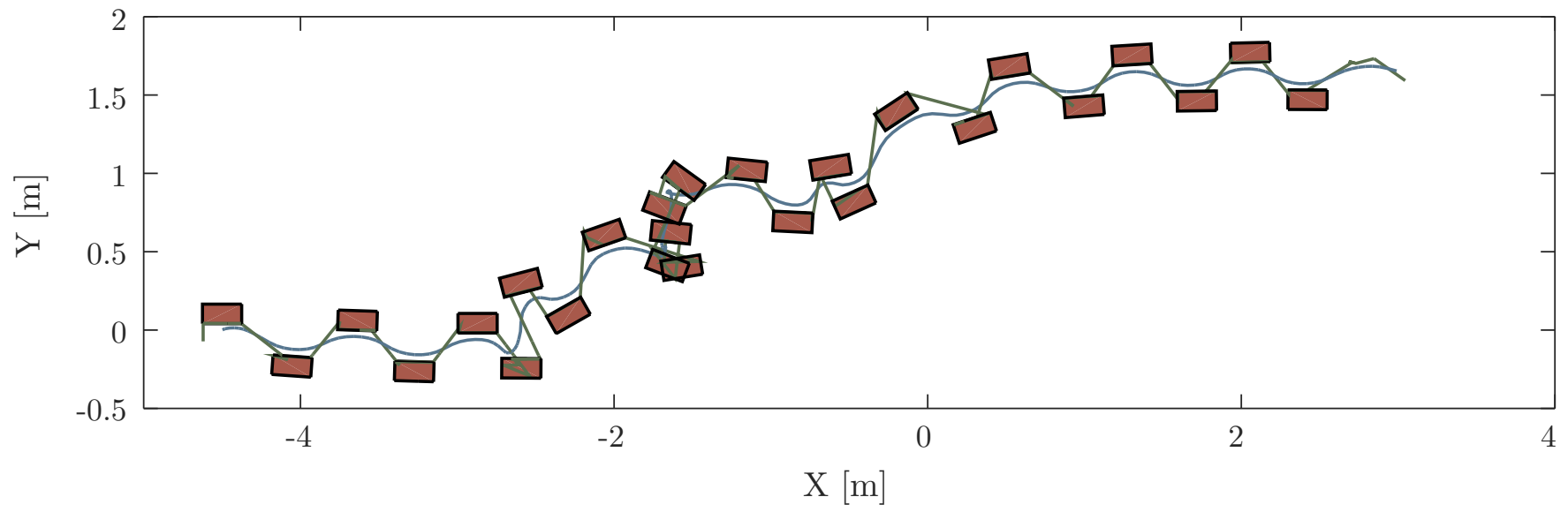

Fig. 4: Adapting the rotation of the feet of the robot while walking in a crowd. The robot walks to the right and the crowd walks in the opposite direction. The figure shows the trajectory of the feet over the course of the simulation.

\section{REFERENCES}

[1] G. Arechavaleta, J.-P. Laumond, H. Hicheur, and A. Berthoz, "An optimality principle governing human walking," IEEE Transactions on Robotics, vol. 24, no. 1, pp. 5-14, feb 2008.

[2] K. Mombaur, A. Truong, and J.-P. Laumond, "From human to humanoid locomotion-an inverse optimal control approach," $A u$ tonomous Robots, vol. 28, no. 3, pp. 369-383, dec 2009.

[3] M. Yagi and V. Lumelsky, "Synthesis of turning pattern trajectories for a biped robot in a scene with obstacles," in Proceedings. 2000 IEEE/RSJ International Conference on Intelligent Robots and Systems (IROS 2000) (Cat. No.00CH37113). IEEE.

[4] S. Aoi, K. Tsuchiya, and K. Tsujita, "Turning control of a biped locomotion robot using nonlinear oscillators," in IEEE International Conference on Robotics and Automation, 2004. Proceedings. ICRA 2004. IEEE, 2004

[5] S. Behnke, "Online trajectory generation for omnidirectional biped walking," in Proceedings 2006 IEEE International Conference on Robotics and Automation, 2006. ICRA 2006. IEEE.

[6] J. Chestnutt, M. Lau, G. Cheung, J. Kuffner, J. Hodgins, and T. Kanade, "Footstep planning for the honda asimo humanoid," Proceedings of the 2005 IEEE International Conference on Robotics and Automation, 2005.

[7] J. Chestnutt, P. Michel, J. Kuffner, and T. Kanade, "Locomotion among dynamic obstacles for the honda ASIMO," in 2007 IEEE/RSJ International Conference on Intelligent Robots and Systems. IEEE, oct 2007.

[8] N. Perrin, O. Stasse, L. Baudouin, F. Lamiraux, and E. Yoshida, "Fast humanoid robot collision-free footstep planning using swept volume approximations', robotics," IEEE Transactions on, p. 439, 2012.

[9] R. Deits and R. Tedrake, "Footstep planning on uneven terrain with mixed-integer convex optimization," 2014 IEEE-RAS International Conference on Humanoid Robots, Nov 2014.

[10] D. Mayne, J. Rawlings, C. Rao, and P. Scokaert, "Constrained model predictive control: Stability and optimality," Automatica, vol. 36, no. 6, pp. $789-814,2000$.

[11] P.-B. Wieber, "Trajectory free linear model predictive control for stable walking in the presence of strong perturbations," in IEEERAS International Conference on Humanoid Robots, Humanoids'06, December, 2006. Genova, Italie: IEEE, Dec. 2006, pp. 137-142.

[12] T. Takenaka, T. Matsumoto, and T. Yoshiike, "Real time motion generation and control for biped robot -1st report: Walking gait pattern generation-," in Intelligent Robots and Systems, 2009. IROS 2009. IEEE/RSJ International Conference on, oct. 2009, pp. 1084 -1091.

[13] R. Tajima, D. Honda, and K. Suga, "Fast running experiments involving a humanoid robot," in Robotics and Automation, 2009. ICRA '09. IEEE International Conference on, May, pp. 1571-1576.

[14] M. Morisawa, K. Harada, S. Kajita, S. Nakaoka, K. Fujiwara, F. Kanehiro, K. Kaneko, and H. Hirukawa, "Experimentation of humanoid walking allowing immediate modification of foot place based on ana- lytical solution," in Robotics and Automation, 2007 IEEE International Conference on, April 2007, pp. 3989-3994.

[15] A. Herdt, N. Perrin, and P.-B. Wieber, "Walking without thinking about it," in 2010 IEEE/RSJ International Conference on Intelligent Robots and Systems. IEEE, oct 2010.

[16] D. D. Simone, N. Scianca, P. Ferrari, L. Lanari, and G. Oriolo, "MPCbased humanoid pursuit-evasion in the presence of obstacles," in 2017 IEEE/RSJ International Conference on Intelligent Robots and Systems (IROS). IEEE, sep 2017.

[17] M. Naveau, M. Kudruss, O. Stasse, C. Kirches, K. Mombaur, and $P$. Soueres, "A reactive walking pattern generator based on nonlinear model predictive control," IEEE Robotics and Automation Letters, vol. 2, no. 1, pp. 10-17, jan 2017.

[18] N. Bohórquez and P.-B. Wieber, "Adaptive step duration in biped walking: a robust approach to nonlinear constraints," in IEEE RAS International Conference on Humanoid Robots 2017, Birmingham, United Kingdom, Nov. 2017.

[19] C. Brasseur, A. Sherikov, C. Collette, D. Dimitrov, and P.-B. Wieber, "A robust linear MPC approach to online generation of $3 \mathrm{~d}$ biped walking motion," in 2015 IEEE-RAS 15th International Conference on Humanoid Robots (Humanoids). Institute of Electrical \& Electronics Engineers (IEEE), nov 2015.

[20] D. Serra, C. Brasseur, A. Sherikov, D. Dimitrov, and P.-B. Wieber, "A Newton method with always feasible iterates for Nonlinear Model Predictive Control of walking in a multi-contact situation," in IEEE-RAS 2016 - International Conference on Humanoid Robots (Humanoids). Cancun, Mexico: IEEE, Nov. 2016, pp. 932-937.

[21] A. Aboudonia, N. Scianca, D. D. Simone, L. Lanari, and G. Oriolo, "Humanoid gait generation for walk-to locomotion using singlestage MPC," in 2017 IEEE-RAS 17th International Conference on Humanoid Robotics (Humanoids). IEEE, nov 2017.

[22] S. Kajita, F. Kanehiro, K. Kaneko, K. Fujiwara, K. Harada, K. Yokoi, and $\mathrm{H}$. Hirukawa, "Biped walking pattern generation by using preview control of zero-moment point," in Robotics and Automation, 2003. Proceedings. ICRA '03. IEEE International Conference on, vol. 2, Sept 2003, pp. 1620-1626 vol.2.

[23] J. Englsberger, C. Ott, M. Roa, A. Albu-Schaffer, and G. Hirzinger, "Bipedal walking control based on capture point dynamics," in Intelligent Robots and Systems (IROS), 2011 IEEE/RSJ International Conference on, sept. 2011, pp. $4420-4427$.

[24] N. Bohórquez, A. Sherikov, D. Dimitrov, and P.-B. Wieber, "Safe navigation strategies for a biped robot walking in a crowd," in IEEERAS International Conference on Humanoid Robots (Humanoids). Cancun, Mexico: IEEE, Nov. 2016, pp. 379-386.

[25] T. Koolen, T. de Boer, J. Rebula, A. Goswami, and J. Pratt, "Capturability-based analysis and control of legged locomotion, part 1: Theory and application to three simple gait models," The International Journal of Robotics Research, vol. 31, no. 9, pp. 1094-1113, 2012. 

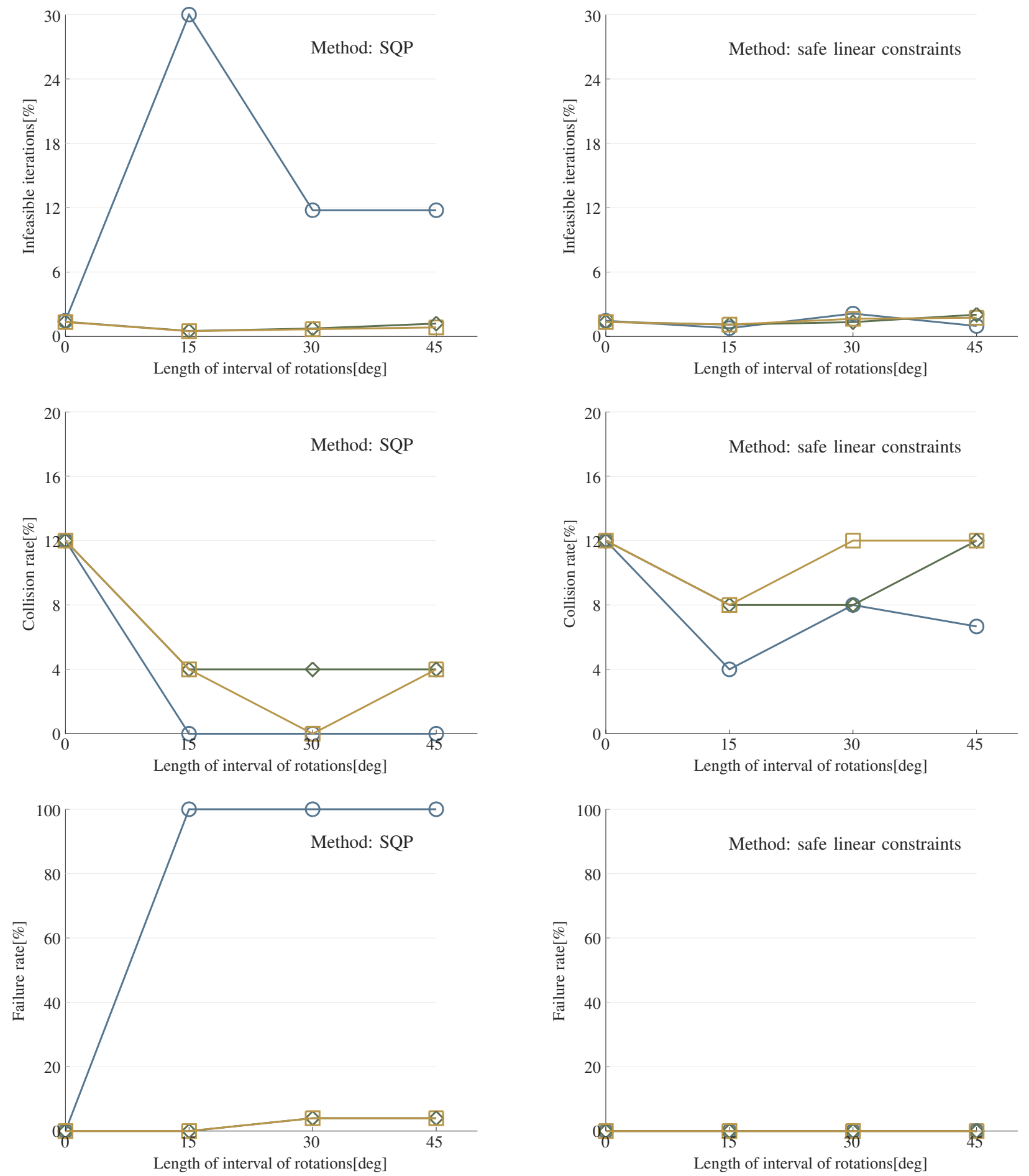

\section{$\vartheta_{1 \text { iteration }} \nabla_{3}$ iterations $\square 5$ iterations}

Fig. 5: Comparison between SQP and the method of safe linear constraints. The infeasible iterations are the proportion of the total number of Newton iterates that were infeasible. The collision rate indicates the proportion of simulations that ended in a previewed collision. The failure rate indicates the proportion of simulations that ended in a nonpreviewed kinematic or dynamic failure. 